\title{
Synthesis and properties of a novel UV-curable waterborne hyperbranched polyurethane
}

\author{
Xufeng Lin, Shouyi Zhang, Jun Qian
}

(C) The Author(s) 2013. This article is published with open access at Springerlink.com

\begin{abstract}
A series of ultraviolet (UV)-curable waterborne hyperbranched polyurethane dispersions (WHPUDs) have been successfully synthesized by modifying the hyperbranched polyester $\mathrm{H} 10$, which was prepared using pentaerythritol as a core molecule and dimethylolpropionic acid as monomers, with succinic anhydride, toluene diisocyanate (TDI), and hydroxypropyl acrylate (HPA). The H10 was characterized by ${ }^{1} \mathrm{H}$ nuclear magnetic resonance spectroscopy and matrix-assisted laser desorption/ionization time of flight mass spectrometry. The properties of the WHPUDs with different content of succinic anhydride and TDI-HPA have been investigated by measuring the stability, the particle size, and the rheological behavior. The effects of the content of succinic anhydride and TDI-HPA were studied in terms of UV-curing rate, water resistance, and thermogravimetric behaviors of WHPUD coatings. The WHPUDs showed good appearance, particle size, viscosity, and storage stability. The WHPUD films showed superior photosensitivity and the percent conversion of $\mathrm{C}=\mathrm{C}$ bonds reached about $80 \%$ when the radiation time was $50 \mathrm{~s}$. Moreover, the UV-cured films had good water resistance and thermostability, which can benefit a waterborne polyurethane resin for waterborne coatings.
\end{abstract}

Keywords UV-curable, Waterborne, Hyperbranched, Polyurethane

X. Lin, S. Zhang, J. Qian ( $\varangle)$

Key Laboratory for Ultrafine Materials of Ministry of

Education, School of Materials Science and Engineering,

East China University of Science and Technology,

Shanghai 200237, People's Republic of China

e-mail: qianjun@ecust.edu.cn

\section{Introduction}

Ultraviolet (UV) radiation is a well-accepted technology for the fast curing of polymeric materials, whereas waterborne coatings are new safe materials that can reduce environmental pollution. Recently, waterborne coatings using UV-curing technology have gained increasing interest because they can decrease air pollution, reduce the risk of fire, improve aspects of occupational health and safety, lower energy consumption, and have high curing speed. ${ }^{1-3}$

Polyurethanes have been popularly applied in organic coatings for their prominent performance. However, ordinary polyurethanes are difficult to disperse in water. Therefore, a special modification on structures is necessary for these oligomers to be dispersible in water. This is normally done by incorporating a small amount of ionic pendant groups. However, the waterborne coatings have also been limited by several problems such as low solid content and low water resistance. ${ }^{4,5}$ To overcome those disadvantages, waterborne polyurethanes have been developed rapidly in recent years. Hyperbranched polymer is one of the most important dendritic polymers synthesized by a relatively new method with relatively low cost. Because of their unique structures, hyperbranched polymers in some cases exhibit properties that are quite different from those of linear polymers. ${ }^{6-8}$ For example, hyperbranched polymers are known to exhibit a very low degree of chain entanglement and typically have relatively low viscosities even at high molecular weights, which is a benefit to coating applications. Recently, the hyperbranched polymers have been widely used to synthesize PU and other materials. $^{9-12}$

The hyperbranched polyester $\mathrm{H} 10$ can be prepared using pentaerythritol (PEL) as a core molecule and dimethylolpropionic acid (DMPA) as monomers, and the UV-curable, waterborne dispersions can be 
prepared by modifying some of the hydroxyl groups of $\mathrm{H} 10$ as a polyol to acidic groups and capping the remaining hydroxyl groups with diisocyanate and acrylic functionalities, such as toluene diisocyanate (TDI) and hydroxypropyl acrylate (HPA), respectively, as shown in Fig. $1 .{ }^{13-15}$ The present paper describes the synthesis and structure-property relationship of a new UV-curable, waterborne, hyperbranched ionomer based on $\mathrm{H} 10$ that was characterized by ${ }^{1} \mathrm{H}$ nuclear magnetic resonance $\left({ }^{1} \mathrm{H}\right.$ NMR) spectroscopy, and matrix-assisted laser desorption/ionization time of flight mass spectrometry (MALDI-TOF MS). A variety of UV-curable, waterborne dispersions have been synthesized with different content of succinic anhydride and TDI-HPA in H10-based prepolymer. The properties of waterborne hyperbranched polyurethane dispersion (WHPUD), such as appearance, storage stability, viscosity, and particle size, have been investigated and the water resistance and thermal properties of UV-cured films were determined. In addition, Fourier transform infrared spectroscopy (FTIR) was used to characterize the structure and study the UV-curing rate of WHPUDs.

\section{Experimental}

\section{Materials}

DMPA and TDI were purchased from J\&K Scientific Ltd., China, and used without further purification. $p$-Toluenesulfonic acid ( $p$-TSA), 1,4-dioxane, stannous chloride dihydrate $\left(\mathrm{SnCl}_{2} \cdot 2 \mathrm{H}_{2} \mathrm{O}\right)$, HPA, succinic anhydride, and triethylamine (TEA) were purchased from Shanghai Linfeng Chemical Co., China. Di- $n$-butyltindilaurate (DBTDL), PEL, and $p$-hydroxyanisole were purchased from Shanghai Guoyao Chemical Co., China. 2-Hydroxyl-2-methyl-1phenyl-1-propanone (Darocur 1173) was supplied by Ciba Co., Switzerland, and used as a photoinitiator.

All the above materials were used as-received without further purification except HPA and 1,4dioxane, which were dried over $\mathrm{CaH}_{2}$ and distilled.
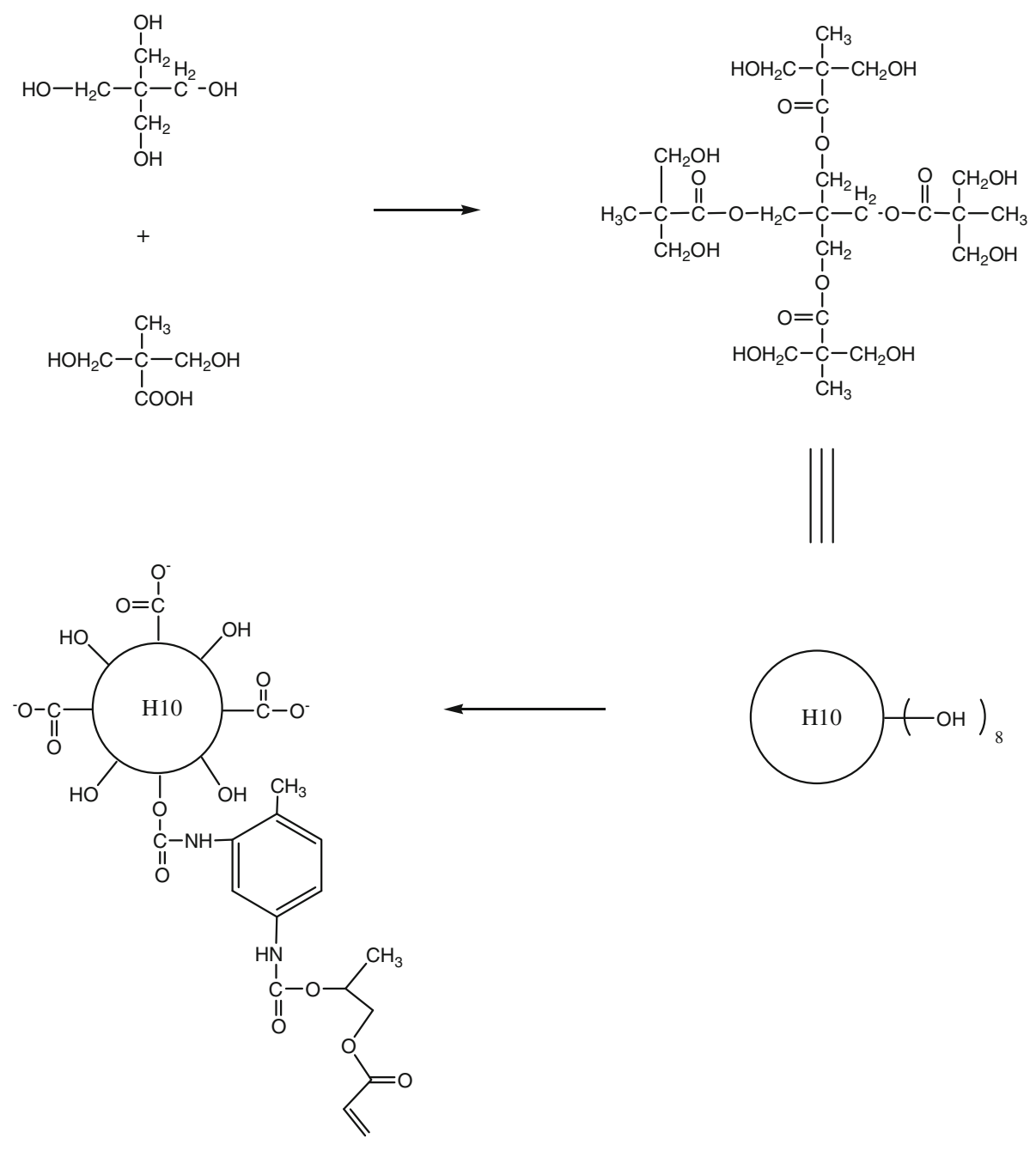

Fig. 1: Synthesis of H10 and UV-curable WHPUD 


\section{Synthesis}

\section{H10 synthesis}

The synthesis was carried out in a $250 \mathrm{~mL}$ threenecked, round-bottomed flask equipped with a mechanical stirrer, thermometer, and condenser with a drying tube. The reaction temperature was controlled between 140 and $160^{\circ} \mathrm{C}$, and $p$-TSA was added as catalyst. A resin with, theoretically, eight hydroxyl groups was yielded after evaporating the small molecule of water, as shown in Fig. $1 .^{16-18}$

\section{PU prepolymer synthesis}

A schematic presentation for the reaction procedure is shown in Fig. 2. The synthesis was carried out in a $250 \mathrm{~mL}$ four-necked, round-bottomed flask equipped with a mechanical stirrer, thermometer, condenser with a drying tube, and $\mathrm{N}_{2}$ gas was aspirated for $10 \mathrm{~min}$ to eliminate residual moisture. The reaction temperature was controlled by using a constant-temperature oil bath. In the first step, $23.6 \mathrm{~g}(39.24 \mathrm{mmol}, 313.86 \mathrm{mmol}$ hydroxyl groups) hyperbranched aliphatic polyester $\mathrm{H} 10$ was dissolved in 1,4-dioxane, and then $8.57 \mathrm{~g}$ (85.6 mmol) succinic anhydride and a catalytic amount of $\mathrm{SnCl}_{2} \cdot 2 \mathrm{H}_{2} \mathrm{O}$ were added. The reaction was conducted at $100^{\circ} \mathrm{C}$ to insure an acceptable rate of reaction until the anhydride peak at $1786 \mathrm{~cm}^{-1}$ in the FTIR spectrum disappeared, as shown in Fig. 3a. Finally, 1,4dioxane was evaporated to obtain a light yellow, highly viscous resin that has, theoretically, three hydroxyl groups conjugated to carboxyl groups. The first step was performed at a relatively low temperature $\left(100^{\circ} \mathrm{C}\right)$ to suppress unwanted side reactions such as etherification and transesterification.

In the second step, $2.484 \mathrm{~g}(14.26 \mathrm{mmol})$ TDI and $15 \mathrm{~mL}$ of 1,4-dioxane were poured into a $250 \mathrm{~mL}$ fournecked, round-bottomed flask equipped with a mechanical stirrer, thermometer, and dropping funnel, and $\mathrm{N}_{2}$ gas was aspirated for $10 \mathrm{~min}$ to eliminate residual moisture. After dissolving $0.1 \mathrm{wt} \%$ DBTDL, $0.1 \mathrm{wt} \%$-hydroxyanisole, and $2.06 \mathrm{~g}(15.85 \mathrm{mmol})$ HPA in $10 \mathrm{~mL}$ dioxane, the mixture was dropped slowly into the flask under efficient stirring at $0^{\circ} \mathrm{C}$ and kept for another $1 \mathrm{~h}$. The temperature was then increased to $30^{\circ} \mathrm{C}$ and maintained for $4 \mathrm{~h}$. Half of $\mathrm{NCO}$ groups remained, as shown in Fig. $3 \mathrm{~b}$. The change of isocyanate $(\mathrm{NCO})$ value during the reaction was determined by the di- $n$-butylamine back titration method to find out the end point of this step.

In the third step, the prepolymer synthesized in the first step, which was cooled to $60^{\circ} \mathrm{C}$, was added dropwise into the above reaction vessel containing TDI-terminated HPA by the dropping funnel and stirred vigorously at $40^{\circ} \mathrm{C}$ until the peak at $2270 \mathrm{~cm}^{-1}$ for $\mathrm{NCO}$ groups completely disappeared, as shown in Fig. 3c. The end point was also determined by the change of NCO value by using the di- $n$-butylamine back titration method.

\section{Neutralization of the prepolymer}

Finally, the residual $-\mathrm{COOH}$ were neutralized with an appropriate amount of TEA $(8.66 \mathrm{~g}, 85.6 \mathrm{mmol})$. The temperature was maintained at $40^{\circ} \mathrm{C}$ for $2 \mathrm{~h}$ to insure completion of the reaction.

\section{Dispersion formation}

After neutralization, the waterborne, hyperbranched polyurethane dispersion was obtained by evaporating the 1,4-dioxane under vacuum, dispersed by adding deionized water to the prepolymer at $35-40^{\circ} \mathrm{C}$. The stirring speed was controlled in 1000-1200 rpm and the dispersion was prepared at $30 \%$ solid content.

For comparison, a series of waterborne, hyperbranched polyurethane dispersions have been prepared. Therefore, in the first step, the stoichiometry for the modification of hydroxyl groups into carboxylic acid groups and acrylate groups was adjusted as shown in Table 1.

\section{Measurements}

FTIR spectra were recorded on a NICOLET 5700 infrared spectrometer by averaging 32 scans using a $\mathrm{KBr}$ disk. The ${ }^{1} \mathrm{H}$ NMR spectra were recorded on a Bruker Avance 500 spectrometer and the chemical shifts were referenced to the residual proton signals of solvent. The molecular weight was determined by an Applied Biosystems Voyager 4418 MALDI-TOF MS spectrometer.

The stability of WHPUD was determined by the centrifugal settling method in the speed of $3000 \mathrm{r} / \mathrm{min}$. If it was still stable after $15 \mathrm{~min}$, it could be determined that the emulsion had 6 months storage stationary phase. The average particle sizes and the distribution of WHPUDs were measured by the laser light scattering method. The viscosity of WHPUDs was measured by a Brookfield-DV-II+ viscometer with $\mathrm{LV}-1$ spindle at $25^{\circ} \mathrm{C}$. The rheological behavior of the final products was determined with variable shear rates.

The UV-curing properties of the WHPUD films were monitored by FTIR. The films were exposed to $365 \mathrm{~nm}$ UV light of $23 \mathrm{mw} / \mathrm{cm}^{2}$ and the distance of the sample to the focal point of the UV lamp was $10 \mathrm{~cm}$.

Thermogravimetric (TG) analysis was carried out with a TG-60 thermoanalyzer (Schimadu Co., Japan). Approximately $5 \mathrm{mg}$ of each sample was used at a heating rate of $20^{\circ} \mathrm{C} / \mathrm{min}$ under $\mathrm{N}_{2}$ from 30 to $650^{\circ} \mathrm{C}$.

The water absorption behavior of UV-cured films was measured at $25^{\circ} \mathrm{C}$ and $100 \%$ relative humidity. 

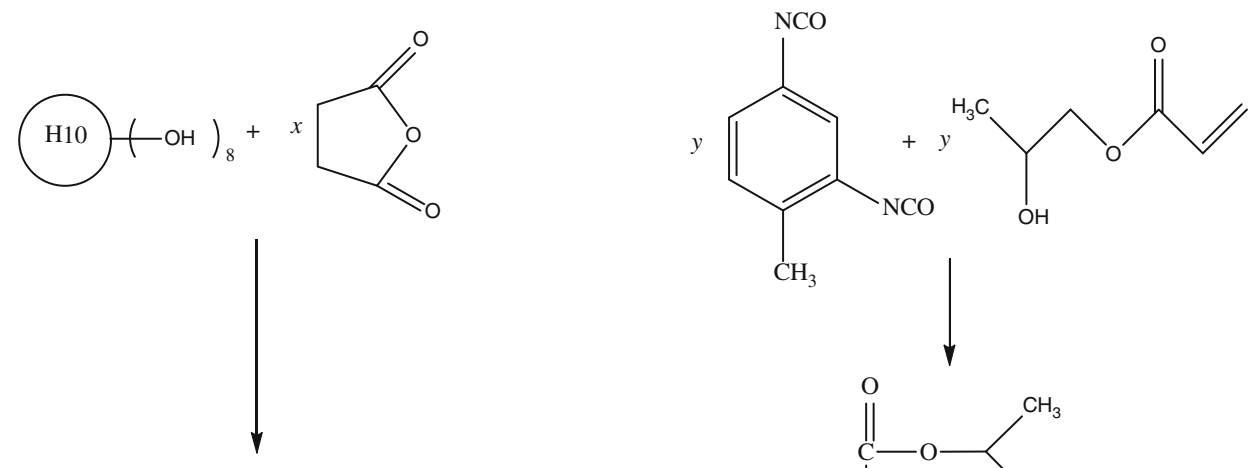

$$
\left(\mathrm{H} 10 \int_{(\mathrm{COOH})}^{(\mathrm{OH})^{8-x}}\right.
$$

$y$<smiles>C=CC(=O)OCC(C)OC(=O)Nc1ccc(C)c([N+](=O)[O-])c1</smiles>

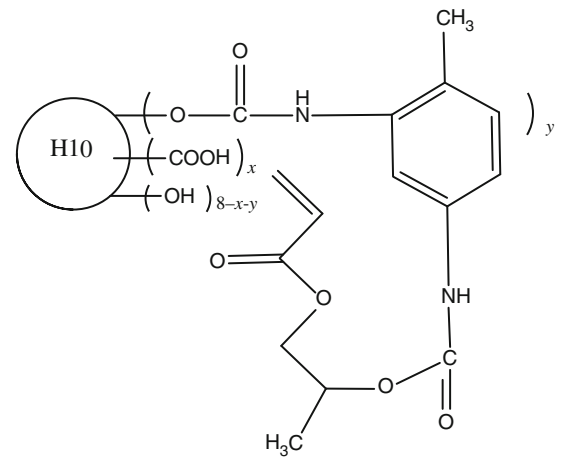<smiles>CCN(CC)CC</smiles>

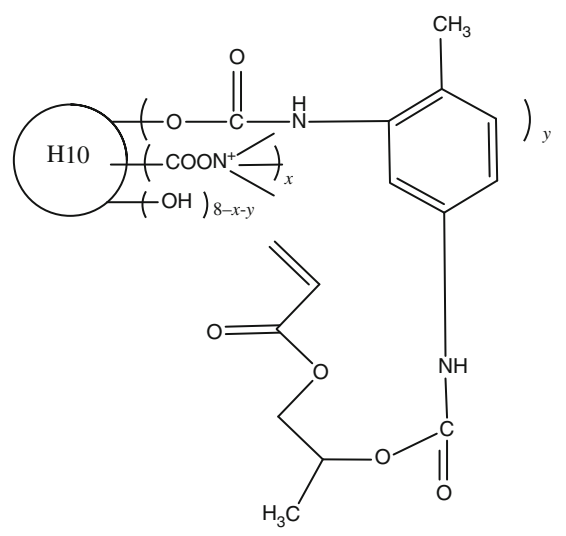

Fig. 2: Schematic description of the synthesis for WHPUD 


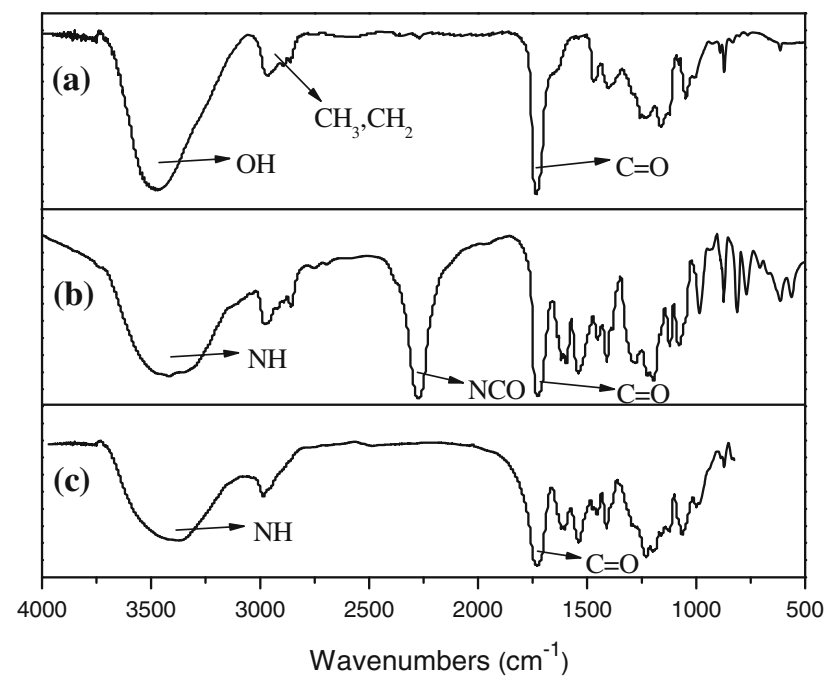

Fig. 3: FTIR spectra comparison of waterborne H10 (a), PU prepolymer (b), and WHPUD (c)

Table 1: Sample designation and stoichiometric ratio of TDI-HPA and -COOH for the preparation of WHPUDs

\begin{tabular}{lccc} 
Samples & \multicolumn{3}{c}{$\begin{array}{l}\text { Stoichiometric ratio of }-\mathrm{COOH}: T D I- \\
\text { HPA }=x: y, n=x+y+N_{\text {remained } \mathrm{OH}}\end{array}$} \\
\cline { 2 - 4 } & $x$ & $y$ & $n-x-y$ \\
\hline WHPUD 2.5-1.0 & 2.5 & 1.0 & 4.5 \\
WHPUD 3.5-1.0 & 3.5 & 1.0 & 3.5 \\
WHPUD 3.0-1.0 & 3.0 & 1.0 & 4.0 \\
WHPUD 3.0-0.5 & 3.0 & 0.5 & 4.5 \\
WHPUD 3.0-1.5 & 3.0 & 1.5 & 3.5 \\
\hline
\end{tabular}

The films were first dried in a vacuum oven at $100^{\circ} \mathrm{C}$ for $12 \mathrm{~h}$. Then the membrane was kept in a humidified chamber with deionized water and the weight change of the films was recorded by using the thin film diffusion analyzer (model D-200; CAHN Instruments, Inc.) until no further water uptake took place. The water uptake $(S)$ was calculated using the following equation:

$S=\left(m_{2}-m_{1}\right) / m_{1} \times 100 \%$,

where $m_{1}$ and $m_{2}$ are the sample weight at the dried state and the sample weight at the saturated state, respectively.

\section{Preparation of $\boldsymbol{U} V$-cured films}

WHPUDs were first mixed with 2 wt \% Darocur 1173, and then the mixture was coated on a tin plate by an

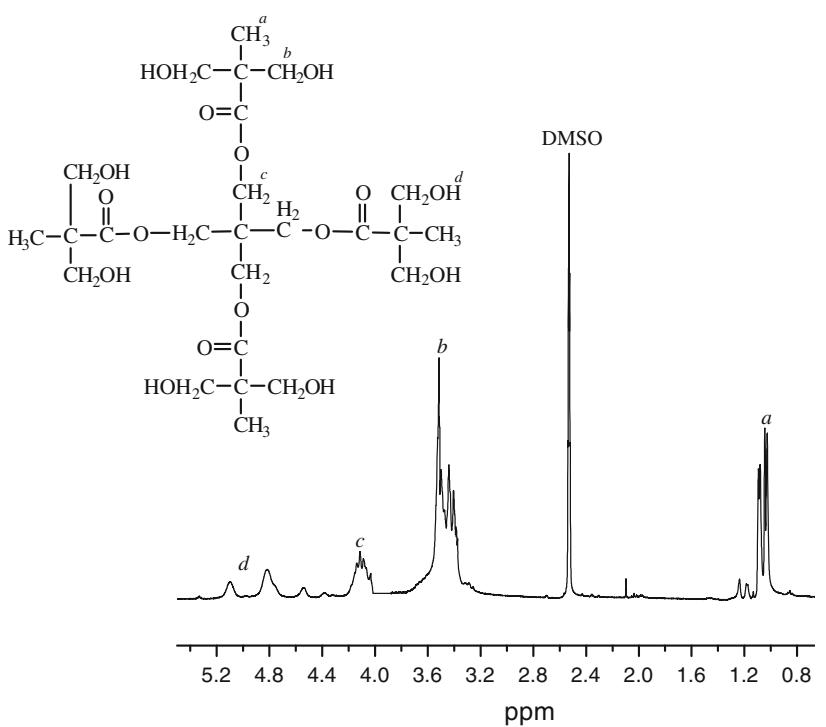

Fig. 4: ${ }^{1} \mathrm{H}$ NMR spectra comparison of hyperbranched polyester $\mathrm{H} 10$

applicator with a $100 \mu \mathrm{m}$ gap, and cured for $60 \mathrm{~s}$ by exposing it to a UV lamp $\left(365 \mathrm{~nm}, 23 \mathrm{mw} / \mathrm{cm}^{2}\right)$.

\section{Results and discussion \\ Characterizations of $\mathrm{H10}$}

In the experiment, the $\mathrm{H} 10$ was prepared using PEL as a core molecule and DMPA as monomers, and it was characterized by ${ }^{1} \mathrm{H}$ NMR and MALDI-TOF MS.

\section{${ }^{1}$ H NMR of H10}

The ${ }^{1} \mathrm{H}$ NMR spectra of the H10 is shown in Fig. 4. Four groups of characteristic peaks are obviously observed, which prove the molecular structure of the H10. The peaks of $a(1.00-1.10 \mathrm{ppm}), b$ (3.35$3.56 \mathrm{ppm})$, and $d(4.65-5.20 \mathrm{ppm})$ are assigned to the hydrogen atom of DMPA. The peaks of $c(4.00$ $4.22 \mathrm{ppm}$ ) are assigned to the hydrogen atom of PEL. The peak at about $2.5 \mathrm{ppm}$ is attributed to the residual hydrogen protons of the solvent (DMSO). All the above analysis confirms the formation of $\mathrm{H} 10$, which is accordance with the ideal structure of H10.

\section{MALDI-TOF MS}

MALDI-TOF MS is regarded as an excellent method for characterizing the molecular weight of H10. The MALDI-TOF MS spectra of $\mathrm{H} 10$ are presented in Fig. 5. The MALDI spectrum shows that the molecular weights of 


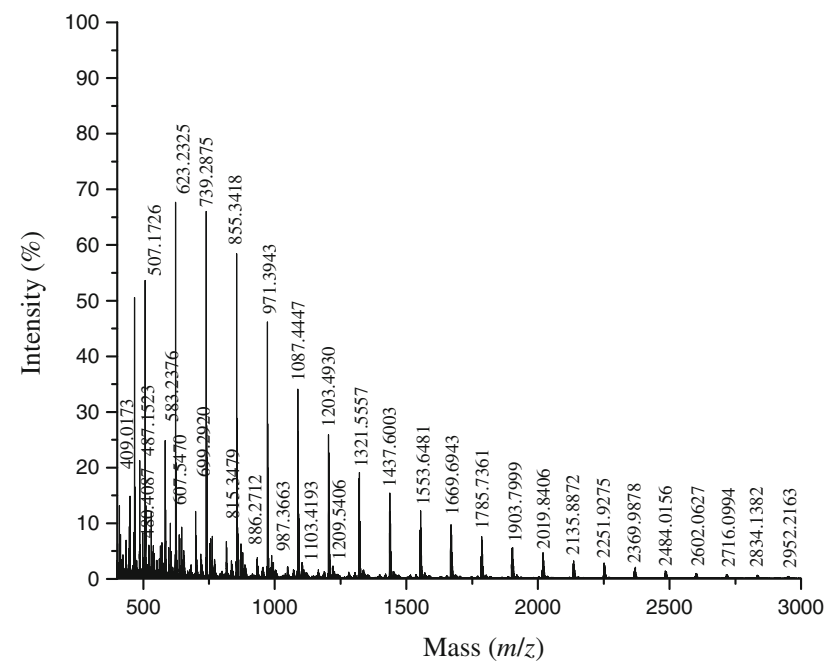

Fig. 5: The molecular weights of the H10

H10 range from 0 to 3000 . And the intense peak is in $507.17-855.34 \mathrm{~g} / \mathrm{mol}$, which contains those calculated by the theory. The theoretical value is $600.25 \mathrm{~g} / \mathrm{mol}$, $(136.15+4 \times 134.03)-4 \times 18.01=600.23 \mathrm{~g} / \mathrm{mol}$.

\section{Appearance, stability, and particle size of WHPUDs}

The appearances, stabilities, and particle sizes of WHPUDs were examined at room temperature. These results are summarized in Table 2. The results in Table 2 show that all WHPUDs have good stabilities except for WHPUD 2.5-10.

The average particle size could be controlled to some extent by such emulsification conditions as stirring speed or dispersing temperature, but it is mostly governed by the concentration of hydrophilic groups. Consequently, the average particle size decreases as the acid content increases. It can be seen in Fig. 6 and Table 2 that the particle sizes follow the order: WHPUD 2.5-1.0 > WHPUD 3.0-1.0 > WHPUD $3.5>1.0$; and WHPUD 3.0-1.5, WHPUD 3.0-0.5 are close to WHPUD 3.0-1.0. The asymptotic decrease of particle size with increasing acid content is due to the stabilizing mechanism of ionomer dispersions. ${ }^{19}$

In this study, the carboxyl content and the TDIHPA content on the average of particle size and stability of WHPUDs were investigated. Only when three or more hydroxyl groups of $\mathrm{H} 10$ have been modified to carboxyl groups do WHPUDs show a better storage stability and a lower particle size.

\section{Rheological behaviors}

The rheological property is a very important factor determining the processibility and end-use property of a polymer material. The chain structure and topology
Table 2: The appearance, storage stability, and size of WHPUDs

\begin{tabular}{|c|c|c|c|}
\hline Samples & Appearance & $\begin{array}{c}\text { Centrifuge (15 min, } \\
3000 \mathrm{r} / \mathrm{min})\end{array}$ & $\begin{array}{l}\text { Size } \\
(\mathrm{nm})\end{array}$ \\
\hline $\begin{array}{r}\text { WHPUD } \\
3.5-1.0\end{array}$ & $\begin{array}{l}\text { White } \\
\text { emulsion }\end{array}$ & Stable & 186 \\
\hline $\begin{array}{r}\text { WHPUD } \\
3.0-1.5\end{array}$ & $\begin{array}{l}\text { White } \\
\text { emulsion }\end{array}$ & Stable & 297 \\
\hline $\begin{array}{l}\text { WHPUD } \\
3.0-1.0\end{array}$ & $\begin{array}{l}\text { White } \\
\text { emulsion }\end{array}$ & Stable & 232 \\
\hline $\begin{array}{l}\text { WHPUD } \\
3.0-0.5\end{array}$ & $\begin{array}{l}\text { White } \\
\text { emulsion }\end{array}$ & Stable & 214 \\
\hline $\begin{array}{r}\text { WHPUD } \\
2.5-1.0\end{array}$ & $\begin{array}{l}\text { White } \\
\text { emulsion }\end{array}$ & Sedimentary (12 min) & 458 \\
\hline
\end{tabular}

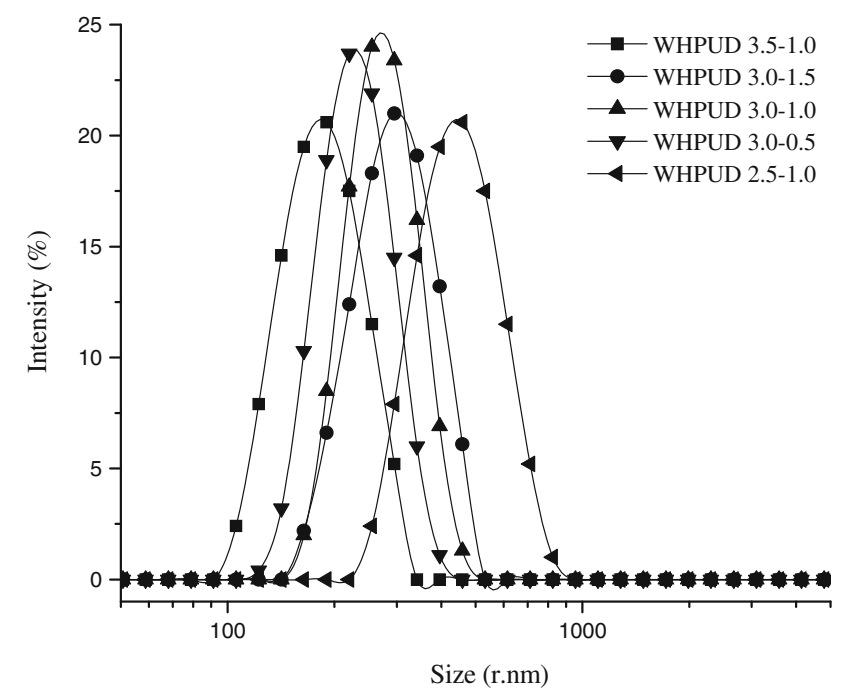

Fig. 6: Size distribution of WHPUDs emulsion

of a polymer determine its rheological properties. The viscosity arises from the interactions between the particles of emulsion. Therefore, many factors, such as molecular architecture, molecular weight, solid content, and dispersion medium, affect the rheological behaviors of emulsion. Moreover, the viscosity of a polymer is related to the dynamic extension and the segment density within the volume of a molecule and intermolecular chain entanglement. As a result of the structures of hyperbranched polymers, which is relatively branched and sphere-like, there will be few entanglements among molecular chains, resulting in a rather lower viscosity. ${ }^{19,20}$

Figure 7 shows the rheological behavior of WHPUD 3.5-1.0, WHPUD 3.0-1.5, WHPUD 3.0-1.0, WHPUD 3.0-0.5, and WHPUD 2.5-1.0. It can be observed that the viscosity for WHPUDs decreases drastically when the shear rate increases from 10 to $30 \mathrm{~min}^{-1}$, whereas it decreases gradually from 30 to $60 \mathrm{~min}^{-1}$. When the shear rate increases, the physical crosslinks between the particles of emulsion are broken down, leading to 
the drastic decrease in viscosity. This implies that WHPUDs exhibit shear thinning or pseudoplastic behavior, which arises in common polymer emulsion. As seen in Fig. 7, WHPUDs exhibit pronounced deviation from Newtonian flow behavior.

The effect of the carboxyl content of WHPUD on the viscosity is also illustrated in Fig. 7. The viscosity of these emulsions follows the order WHPUD 2.5-1.0 < WHPUD 3.0-1.0 < WHPUD 3.5-1.0. As the concentration of ionic groups in the PU molecule increases, the mutual repulsion of the same charges causes the expansion of chains, which may result in increasing the viscosity.

The hard segment of prepolymer also plays an important role in determining the viscosity. The viscosity of WHPUDs emulsion follows the order of WHPUD 3.0-0.5 < WHPUD 3.0-1.0 < WHPUD 3.0-1.5. The chain entanglement and hydrogen bonding
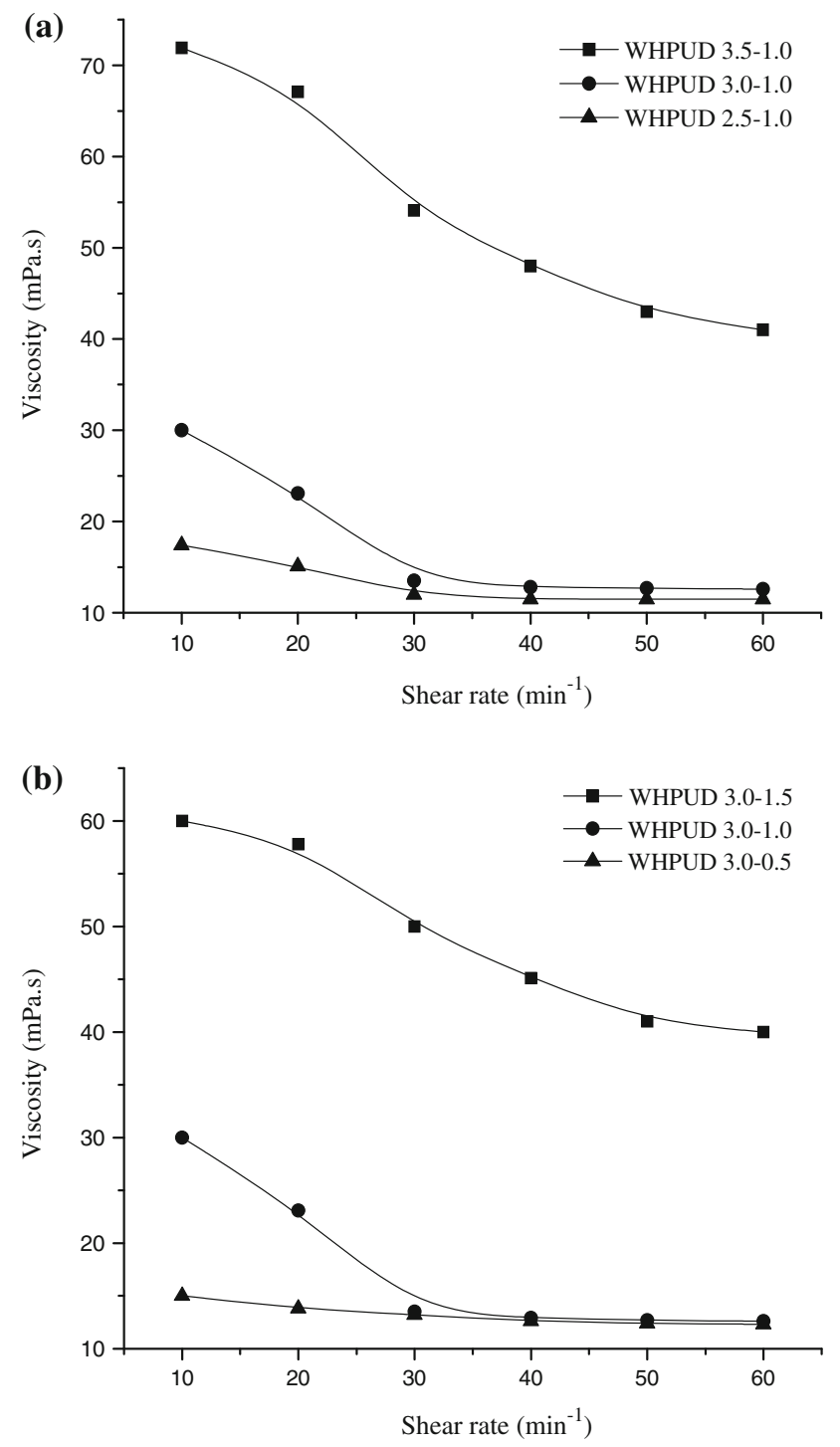

Fig. 7: Viscosity-shear rate of WHPUDs emulsion between urethane and urea linkages can be more rapidly formed in the polymer because of the flexibility of the chains. As the content of prepolymer TDI-HPA increases, the chain entanglement and hydrogen bonding increases, which may result in increasing the viscosity.

\section{UV curing}

The UV-curing properties of the synthesized WHPUDs were measured by FTIR. In order to stimulate the photoinitiator, the film was exposed to $23 \mathrm{~mW} / \mathrm{cm}^{2}$ of $365 \mathrm{~nm}$ light for an increasing amount of time. Figure 8 shows the FTIR spectra of WHPUD $3.0-1.0$ in the absence of photoinitiator at various curing time(s) $(0,10,20,30,40,50)$.

The percent conversion of $\mathrm{C}=\mathrm{C}$ bond is calculated from the decay of the absorption bands of the double bond group $\left(\mathrm{CH}=\mathrm{CH}_{2}\right)$ twisting vibration at $810 \mathrm{~cm}^{-1}$, and the characteristic absorption peak of carbonyl group $(\mathrm{C}=\mathrm{O})$ stretching mode at $1720 \mathrm{~cm}^{-1}$ is used as the internal standard peak by integration of the peak areas. Hence, the double bond conversion (c) after a given exposure time $(t)$ of $\mathrm{CH}=\mathrm{CH}_{2}$ can be readily obtained from the ratio of the FTIR absorbance areas before $\left(A_{0}\right)$ and after UV irradiation $\left(A_{t}\right)$ by equation (2). ${ }^{21-23}$

$c(\%)=\frac{\left(\frac{A_{810}}{A_{1720}}\right)_{0}-\left(\frac{A_{810}}{A_{1720}}\right)_{t}}{\left(\frac{A_{810}}{A_{1720}}\right)_{0}} \times 100$

Figure 9 illustrates the relationship between the double bond conversion (\%) and irradiation time (s). It can be found that, in the initial stage of UV radiation, the percent conversion of $\mathrm{C}=\mathrm{C}$ bond increases quickly with the increase of radiation time. The maximum conversion value of $\mathrm{C}=\mathrm{C}$ bond is about $80 \%$ when exposure time is $50 \mathrm{~s}$. The conversion increases slightly when the radiation time exceeds $30 \mathrm{~s}$, indicating that WHPUDs have superior photosensitivity.

As shown in Fig. 9, five types of WHPUDs with the increase of irradiation time follows the order of WHPUD 3.0-1.5 > WHPUD 3.0-1.0 > WHPUD 3.0-0.5, and the values of $c$ of WHPUD 3.5-1.0 and WHPUD 2.5-1.0 are close to WHPUD 3.0-1.0. The results can be explained with the double bond concentration. In general, the higher concentration of double bonds of the acrylic end group located at the outer layer of the sphere-like structure may lead to higher conversion.

\section{Water resistance}

Waterborne polyurethanes have a large number of hydrophilic terminal groups, so the water resistance of 
waterborne polyurethane is lower than that of solvent polyurethanes. This factor becomes the restriction of the development of waterborne polyurethane. To overcome this disadvantage, the present paper uses the unique hyperbranched polymer (H10) and UV-curing technology to improve the water resistance of waterborne polyurethane. . $^{2,24}$

The water absorption of UV-cured films is shown in Table 3. As seen in Table 3, the water absorption of UV-cured films decreases with increasing double bond concentration (the content of TDI-HPA),WHPUD 3.0-0.5 > WHPUD 3.0-1.0 > WHPUD 3.0-1.5. The films with higher double bond concentration exhibited better water resistance, and this may be ascribed to the higher crosslinking density between crosslinking points after UV curing. In the same crosslinking density, along

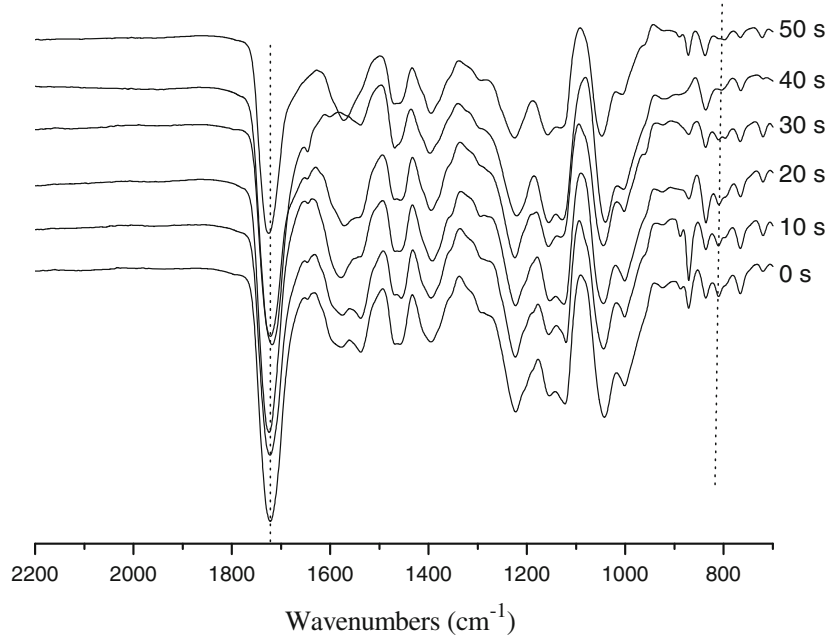

Fig. 8: FTIR spectra of the course of UV curing (WHPUD 3.0-1.0)

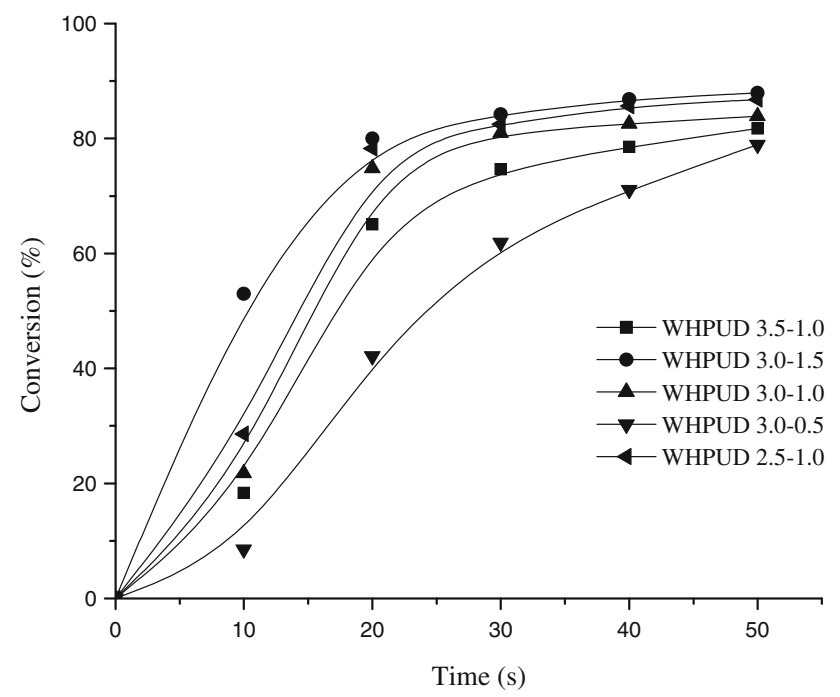

Fig. 9: Conversion of double bond of WHPUDs with the carboxyl content increasing, the water absorption gradually increases, WHPUD 2.5-1.0 < WHPUD 3.0-1.0 < WHPUD 3.5-1.0. The film with fewer hydrophilic groups has a better water resistance.

Therefore, hyperbranched polyurethane has water resistance that is superior to linear polyurethane. In addition, after UV curing, it is able to form a threedimensional chemical bonding between PU polymer molecules, leading to the increasing crosslinking density of the polymer and decreasing free volume of the molecule chain. Thus, the water molecules are difficult

Table 3: The water sorption of WHPUDs UV-cured film

\begin{tabular}{lccc} 
Samples & $m_{1}(\mathrm{~g})$ & $m_{2}(\mathrm{~g})$ & Absorption (\%) \\
\hline WHPUD 3.5-1.0 & 1.092 & 1.464 & 34.1 \\
WHPUD 3.0-1.5 & 0.837 & 0.998 & 19.3 \\
WHPUD 3.0-1.0 & 1.587 & 1.947 & 22.7 \\
WHPUD 3.0-0.5 & 0.967 & 1.234 & 27.6 \\
WHPUD 2.5-1.0 & 2.192 & 2.595 & 18.4 \\
\hline
\end{tabular}
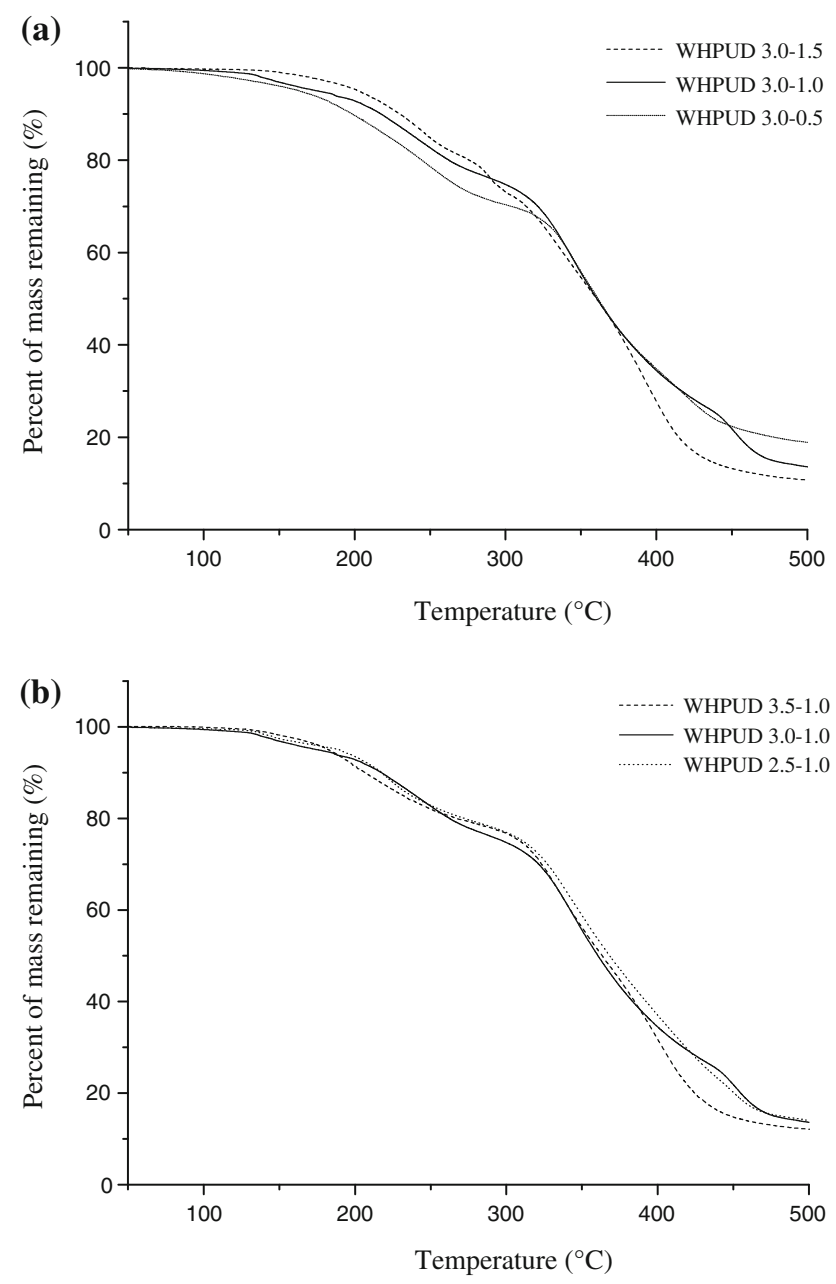

Fig. 10: Thermogravimetric curves of WHPUDs cured films 
Table 4: The degradation temperature at $10 \%$ weight loss

Samples

$10 \%$ weight loss $\left({ }^{\circ} \mathrm{C}\right)$

WHPUD 3.5-1.0

212.50

WHPUD 3.0-1.5

229.74

WHPUD 3.0-1.0

217.44

WHPUD 3.0-0.5

WHPUD 2.5-1.0

198.40

217.34

to penetrate into the films, and therefore the water resistance increases.

\section{Heat resistance}

The heat resistance investigation of polyurethanes allows the determination of proper conditions to obtain high-performance products which are stable and free of undesired by-products. Besides, TG provides a method for accelerating the lifetime testing of polyurethanes so it can be used to predict in-use lifetime.

Earlier work has shown that the thermal degradation initiates at the urethane group of hard segment. Polyurethane has at least two stages of degradation, where the initial stage is mainly due to the decomposition of hard segment. TG curves of UV-cured films are shown in Fig. 10 and Table 4, which present the degradation temperatures at a weight loss of $10 \%$.

As seen in Fig. 10, all samples have a similar process of thermal decomposition. It is obvious that the initial temperature of decomposition and the temperature of complete decomposition for WHPUD films were about 200 and $315^{\circ} \mathrm{C}$.

The degradation in the early stage plays an important role in the overall thermal stability of WHPUDs films. As seen in Table 4, the temperature of $10 \%$ weight loss is WHPUD $3.0-1.5>$ WHPUD $3.0-1.0>$ WHPUD 3.0-0.5, and the temperatures of WHPUD 3.5-1.0 and WHPUD 2.5-1.0 are close to WHPUD 3.0-1.0. The heat resistance of UV-cured films is improved with the increasing of hard segment. Samples with higher hard segment result in films with higher crosslinking density after being cured by UV and the possibility of hydrogen bonding formation, and possess better heat resistance. These results are coincident with the paper reported by Asif et al. ${ }^{25}$

\section{Conclusions}

In conclusion, we have successfully synthesized a series of UV-curable waterborne hyperbranched polyurethane by modifying the hyperbranched polyester $\mathrm{H} 10$, which was prepared using PEL as a core molecule and DMPA as monomers, with succinic anhydride, TDI, and HPA. The rheological behavior of WHPUDs suggested that all WHPUDs belong to pseudoplastic fluids and have a lower viscosity. The investigation of UV-curing kinetics indicates that these WHPUDs films are photosensitive, and that the percent conversion of $\mathrm{C}=\mathrm{C}$ bond reaches about $80 \%$ when the exposure time was $50 \mathrm{~s}$. TG analysis indicated that the heat resistance of the UV-cured films increased with an increase of the content of TDI-HPA, which is attributed to the formation of a highly crosslinked network through UV curing. Moreover, the UV-cured films showed excellent water resistance properties. In the field of overall properties, WHPUD 3.0-1.0 is the best sample with good storage stability, low viscosity, and small particle size. Moreover, the UV-cured film of WHPUD 3.0-1.0 has good water and heat resistance.

Open Access This article is distributed under the terms of the Creative Commons Attribution License which permits any use, distribution, and reproduction in any medium, provided the original author(s) and the source are credited.

\section{References}

1. Ates, S, Aydogan, B, Torun, L, Yagci, Y, "Synthesis and Characterization Oftriptycene Type Crosslinker and Its Use in Photoinduced Curing Applications." Polymer, 51 825-831 (2010)

2. Han, WS, Lin, BP, Yang, H, Zhang, XQ, "Synthesis and Properties of UV-Curable Hyperbranched Polyurethane Acrylate Oligomers Containing Carboxyl Groups." Polym. Bull., 68 1009-1022 (2012)

3. Tasic, S, Bozic, B, Dunjic, B, "Synthesis of New Hyperbranched Urethane-Acrylates and Their Evaluation in UV-Curable Coatings." Prog. Org. Coat., 51 321-328 (2004)

4. Gao, QZ, Li, HQ, Zeng, XR, "Preparation and Characterization of UV-Curable Hyperbranched Polyurethane Acrylate." J. Coat. Technol. Res., 8 (1) 61-66 (2011)

5. Han, WS, Lin, BP, Zhou, YD, Song, JG, "Synthesis and Properties of UV-Curable Hyperbranched Polyurethane Acrylate Oligomers Containing Photoinitiator." Polym. Bull., 68 729-743 (2012)

6. Gregorowicza, J, Fras, Z, Parzuchowski, P, Rokicki, G, Kusznerczukb, M, Dziewulski, S, "Phase Behaviour of Hyperbranched Polyesters and Polyethers with Modified Terminal OH Groups in Supercritical Solvents." J. Supercrit. Fluid., 55 786-796 (2010)

7. Zhao, XF, Liu, LX, Dai, HX, Ma, CX, Tan, XH, Yu, RH, "Synthesis and Application of Water-Soluble Hyperbranched Poly(ester)s from Maleic Anhydride and Glycerol." J. Appl. Polym. Sci., 113 3376-3381 (2009)

8. van Benthem Rolf, ATM, "Novel Hyperbranched Resins for Coating Applications." Prog. Org. Coat., 40 203-214 (2000)

9. Cheng, XE, Huang, ZG, Liu, JH, Shi, WF, "Synthesis and Properties of Semicrystalline Hyperbranched Poly(esteramide) Grafted with Long Alkyl Chains Used for UV-Curable Powder Coatings." Prog. Org. Coat., 59 284290 (2007)

10. Foix, D, Fernández-Francos, X, Ramis, X, Serra, A, Sangermano, M, "New Pegylated Hyperbranched Polyester as Chemical Modifier of Epoxy Resins in UV Cationic Photocuring."' React. Funct. Polym., 71 417-424 (2011) 
11. Morgan, S, Ye, ZB, Zhang, KJ, Subramanian, R, “One-Pot Synthesis of Hyperbranched Polyethylenes Tethered with Pendant Acryloyl Functionalities by Chain Walking Copolymerizations." Macromol. Chem. Phys., 209 2232-2240 (2008)

12. Deka, H, Karak, N, "Bio-based Hyperbranched Polyurethanes for Surface Coating Applications." Prog. Org. Coat., 66 192-198 (2009)

13. Miao, H, Cheng, LL, Shi, WF, "Fluorinated Hyperbranched Polyester Acrylate Used as an Additive for UV Curing Coatings." Prog. Org. Coat., 65 71-76 (2009)

14. Florian, P, Jena, KK, Allauddin, S, Narayan, R, Raju, KVSN, "Preparation and Characterization of Waterborne Hyperbranched Polyurethane-Urea and Their Hybrid Coatings." Ind. Eng. Chem. Res., 49 4517-4527 (2010)

15. Maji, PK, Guchhait, PK, Bhowmick, AK, "Effect of the Microstructure of a Hyperbranched Polymer and Nanoclay Loading on the Morphology and Properties of Novel Polyurethane Nanocomposites." ACS Appl Mater Interfaces, 1 (2) 289-300 (2009)

16. Du, YY, Chen, QL, Shen, L, Xing, YJ, Dai, JJ, "Synthesis and Application of Long-Chain Alkyl Quaternary Ammonium-Functionalized Hyperbranched Polyester." J. Appl. Polym. Sci., 121 2927-2935 (2011)

17. Zhang, XL, "Synthesis and Characterization of Hyperbranched Polyesters Based on Isophthalic Acid and Trimethylolpropane." J. Macromol. Sci. A, 48 128-134 (2011)

18. Murillo, EA, Cardona, A, Lopez, BL, "Rheological Behavior in the Molten State and Solution of Hyperbranched
Polyester of Fourth and Fifth Generation." J. Appl. Polym. Sci., 119 929-935 (2011)

19. Asif, A, Huan, CY, Shi, WF, "Structure-Property Study of Waterborne Polyurethane Acrylate Dispersions Based on Hyperbranched Aliphatic Polyester for UV-Curable Coatings." Colloid Polym. Sci., 283 200-208 (2004)

20. Asif, A, Hu, LH, Shi, WF, "Synthesis, Rheological, and Thermal Properties of Waterborne Hyperbranched Polyurethane Acrylate Dispersions for UV Curable Coatings." Colloid Polym. Sci., 287 1041-1049 (2009)

21. Yin, WH, Zeng, XR, Li, HQ, Hou, YJ, Gao, QZ, "Synthesis, Photopolymerization Kinetics, and Thermal Properties of UVCurable Waterborne Hyperbranched Polyurethane Acrylate Dispersions." J. Coat. Technol. Res., 8 (5) 577-584 (2011)

22. Gao, QZ, Li, HQ, Zeng, XR, "UV-Curing of Hyperbranched Polyurethane Acrylate-Polyurethane Diacrylate/ $\mathrm{SiO}_{2}$ Dispersion and TGA/FTIR Study of Cured Films." J. Cent. South. Univ., 19 63-70 (2012)

23. Wang, SJ, Liu, X, Kong, J, Tian, W, Fan, XD, Xu, H, Lu, JR, "Synthesis and UV Curing Kinetics of Rapidly UV-Curable Hyperbranched Polycarbosiloxanes." Polym. Int., 59 13231330 (2010)

24. Qiao, LG, Shi, WF, "Synthesis and Characterization of Hyperbranched Polyurethane-Benzyltetrazole." Chin. J. Polym. Sci., 6 (29) 670-683 (2011)

25. Asif, A, Hu, LH, Shi, WF, "Synthesis, Rheological, and Thermal Properties of Waterborne Hyperbranched Polyurethane Acrylate Dispersions for UV Curable Coatings." Colloid. Polym. Sci., 287 (9) 1041-1049 (2009) 